\title{
Hydrodynamic theory of chiral angular momentum generation in metals
}

\author{
Hiroshi Funaki 1 and Gen Tatara \\ RIKEN Center for Emergent Matter Science (CEMS), and RIKEN Cluster for Pioneering Research (CPR), \\ 2-1 Hirosawa, Wako, Saitama, 351-0198 Japan
}

(Received 7 October 2020; accepted 28 April 2021; published 28 May 2021)

\begin{abstract}
We present a hydrodynamic theory to describe a chiral electron system with a Weyl spin-orbit interaction on a field-theoretic basis. Evaluating the momentum flux density as a linear response to a driving electric field, we derive an equation of motion for the orbital angular momentum. It is shown that the chiral nature leads to a dynamic bulk angular momentum generation by inducing a global torque as a response to the applied field. The steady-state angular momentum is calculated taking into account rotational viscosity.
\end{abstract}

DOI: 10.1103/PhysRevResearch.3.023160

\section{INTRODUCTION}

Hydrodynamic theory is essential in describing fluids at macroscopic scales. Conventional fluids are described by momentum flux tensor $\pi_{i j}$ symmetric with respect to the direction of momentum $i$ and flow $j$ [1]. Recently, fluids consisting of particles with internal degrees of freedom such as polar and chiral natures have drawn considerable attention, as such symmetries lead to unique fluids (chiral fluids and polar fluids) with different symmetries of the momentum flux tensor. For instance, chiral fluids have antisymmetric components of the tensor, which govern the angular momentum dynamics of the fluids, playing essential roles in many biological phenomena [2-4].

Hydrodynamic theory is based on equations representing a continuity of densities of fluid, momentum, and energy at macroscopic scales. The coefficients in the equations are generally treated as phenomenological parameters determined by the symmetry of the system. Even microscopic quantum objects such as electrons in conducting solids are treated as a fluid when viewed at macroscopic scales. The hydrodynamic approach has in fact been applied to study the electron transport properties of solids [5-9]. Conventional fluids are characterized by viscosity, while relaxation force due to scattering by extrinsic scattering is important for electron fluids in disordered metals. The electron fluids are thus categorized into two regimes, a viscous regime and the Ohmic regime realized for $\ell_{\mathrm{v}} \ll \ell$ and $\ell_{\mathrm{v}} \gg \ell$, respectively [10], where $\ell_{\mathrm{v}}$ and $\ell$ are characteristic length scales of viscosity and extrinsic scattering, respectively (Table I). The flow profile in the viscous regime is the Poiseuille flow, while that in the Ohmic limit is a constant determined by the relaxation. The viscous electron flow and the intermediate regime (called the porous regime) have been observed in high-mobility graphene [11]. Hydrodynamic theories of electron fluids in solids have been

Published by the American Physical Society under the terms of the Creative Commons Attribution 4.0 International license. Further distribution of this work must maintain attribution to the author(s) and the published article's title, journal citation, and DOI. presented based on the semiclassical Boltzmann approach $[6,12,13]$ and Berry's phase representation $[14,15]$. In hydrodynamic theories for electrons, coefficients characterizing the fluid can in principle be microscopically determined with use of a linear-response theory with systematic approximations [16-18]. In such microscopic methods, the continuity equation for the density is automatically guaranteed by keeping the gauge invariance, and only the equation for the momentum density needs to be considered.

The aim of this paper is to construct a hydrodynamic theory for describing chiral electron systems in solids on a field-theoretic basis. Considering disordered metals, we discuss the Ohmic (dissipative) regime with relaxation. The chiral nature is introduced by a three-dimensional spin-orbit interaction that is linear in wave vector $\boldsymbol{k}$, called the Weyl spinorbit interaction. Based on a quantum field representation, we derive an equation of motion for the momentum operator, and we identify the momentum flux tensor as a field operator. The expectation value of the tensor is evaluated with use of nonequilibrium Green's functions as a linear response to a driving force, i.e., the applied electric field $\boldsymbol{E}$, resulting in a coarse-grained equation for the momentum density $\boldsymbol{p}$. For the Ohmic fluid, the equation is written in terms of the local current density $\boldsymbol{j}$ with use of a constitutional equation $j_{i}=$ $\left(\sigma_{\mathrm{e}}\right)_{i j} E_{j}$, where the conductivity tensor $\sigma_{e}$ is determined by the linear-response theory on the same footing as the equation for $\boldsymbol{p}$. We obtain, therefore, a hydrodynamic equation in the conventional form with each term evaluated fully quantum mechanically without assuming a classical transport equation. Based on the equation, we demonstrate that a field applied to a chiral fluid induces a bulk torque, resulting in bulk orbital angular momentum generation.

\section{FORMALISM}

The field Hamiltonian we consider is (a caret denotes a field operator)

$$
\hat{H}=\sum_{k} \hat{c}_{k}^{\dagger}\left(\epsilon_{k}+\gamma_{k} \cdot \sigma\right) \hat{c}_{k},
$$


TABLE I. Two regimes of electron fluid, namely a viscous regime and the Ohmic regime, governed by the viscosity with length scale $\ell_{\mathrm{v}}$ and relaxation force with scattering length $\ell$, respectively.

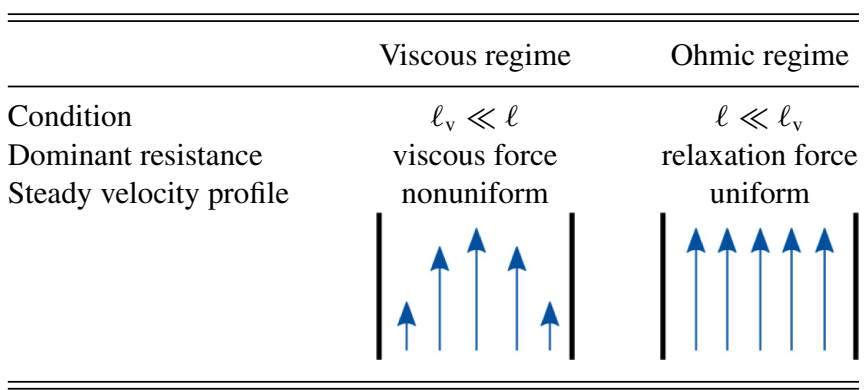

where $\hat{c}$ and $\hat{c}^{\dagger}$ are conduction electron field operators with two spin components, $\sigma$ is a vector of Pauli matrices, and $\epsilon_{k} \equiv$ $\frac{k^{2}}{2 m}-\epsilon_{F}$ is the free-electron energy $\left(\epsilon_{F}\right.$ is the Fermi energy, $m$ is the electron mass). A vector $\gamma_{k} \equiv \lambda \boldsymbol{k}$ represents the Weyl spin-orbit interaction with a coupling constant $\lambda$. The electron velocity operator in the momentum space is

$$
\hat{v}_{i}=\frac{k_{i}}{m}+a_{i}^{\alpha} \sigma_{\alpha},
$$

where

$$
a_{i}^{\alpha}=\frac{d \gamma_{k}^{\alpha}}{d k_{i}}
$$

is the anomalous velocity due to the spin-orbit interaction. The Hamiltonian (1) is allowed in materials that belong to the chiral point groups $O$ and $T$ [19], and indeed it was explicitly derived for B20 compounds that belong to the point group $T$ [20]. Equation (1) assumes that a single Weyl node exists at the Fermi level, and the other(s) lie at high energies. To be precise, in any lattice systems, Weyl nodes appear in a pair [21], and some chiral effects such as the chiral magnetic effect are canceled [22]. However, for most transport properties, cancellation within a pair does not occur when the electron distributions at the nodes differ, as in the case in which a counterpart of the pair lies at a high-energy state as observed in first-principles calculations for trigonal Te and Se [23].

Our hydrodynamic equation is derived by calculating $\dot{\hat{\boldsymbol{p}}}$, the time derivative of the momentum operator, which reads $\dot{\hat{p}}_{i}=\nabla_{j} \hat{\pi}_{i j}$, where $\hat{\pi}_{i j} \equiv \hat{c}^{\dagger} \hat{p}_{i} \hat{v}_{j} \hat{c}$ is the momentum flux tensor operator. We thus obtain a coarse-grained hydrodynamic equation

$$
\dot{p}_{i}=\nabla_{j} \pi_{i j}
$$

for expectation values $p_{i}$ and $\pi_{i j}$ of $\hat{p}_{i}$ and $\hat{\pi}_{i j}$, respectively. Equation (4) obtained as a field average applies to length scales much larger than the electron mean free path. This simple equation is sufficient in most cases to describe the linear regime of the fluid. In conventional fluids, the momentum flux tensor $\pi_{i j}$ has a diagonal part $-P \delta_{i j}$ representing a pressure $P$, and a symmetric part $\eta_{\mathrm{s}}\left(\nabla_{i} v_{j}+\nabla_{j} v_{i}\right)$ linear in both velocity $v_{i}$ and the spatial derivative, representing a viscosity $\eta_{\mathrm{s}}$ [see Eq. (8)] [1].

For fluid dynamics with local velocity $\boldsymbol{v}$, external driving force is necessary. We consider an electric field, represented by a vector potential $\boldsymbol{A}$ as $\boldsymbol{E}=-\dot{\boldsymbol{A}}$. Considering a linearresponse regime, a local fluid velocity $\boldsymbol{v}$ arises as a linear function of the local electric field. As the system of interest is in the weakly driven fluid regime, quadratic terms in $v$ commonly argued in the standard hydrodynamic equations are neglected.

Considering a static limit, the momentum flux tensor at linear order reads $\pi_{i j}=\pi_{i j k} E_{k}$, where the response function is $\pi_{i j k}(\boldsymbol{q})=\lim _{\omega_{0} \rightarrow 0} \frac{i}{\omega_{0}} \int \frac{d \omega}{2 \pi} \frac{e}{V} \sum_{\boldsymbol{k}} \operatorname{tr}\left[k_{i} \hat{\boldsymbol{v}}_{j} G_{\omega, \boldsymbol{k}} \hat{v}_{k} G_{\omega+\omega_{0}, \boldsymbol{k}+\boldsymbol{q}}\right]^{<}$, where $G_{\omega, \boldsymbol{k}}$ is the path-ordered Green's function [24] with angular frequency $\omega$ and wave vector $\boldsymbol{k},<$ denotes the lesser component, tr is a trace over the spin indices, $e$ is the electron charge, and $V$ is the system volume. The wave vector and angular frequency of the external field are $\boldsymbol{q}$ and $\omega_{0}$, respectively, and the static limit $\omega_{0} \rightarrow 0$ is taken at the end of the calculation. Focusing on the dynamically driven flow, the dominant excited contributions at low temperatures read

$$
\pi_{i j k}(\boldsymbol{q})=\frac{e}{V} \sum_{\boldsymbol{k}} \operatorname{tr}\left[k_{i} \hat{v}_{j} G_{\boldsymbol{k}}^{\mathrm{r}} \hat{v}_{k} G_{\boldsymbol{k}+\boldsymbol{q}}^{\mathrm{a}}\right],
$$

where $G_{\boldsymbol{k}}^{\lambda} \equiv G_{\omega=0, \boldsymbol{k}}^{\lambda} \quad(\lambda=\mathrm{a}, \mathrm{r})$, and $G_{\boldsymbol{k}}^{\mathrm{r}}=\frac{1}{-\epsilon_{k}-\gamma_{k} \cdot \sigma+\frac{i}{2 \tau}}$ and $G_{k}^{\mathrm{a}}=\left(G_{\boldsymbol{k}}^{\mathrm{r}}\right)^{*}$ are the retarded and advanced Green's function, respectively. We take into account finite elastic electron lifetime $\tau$ as an imaginary part of the energy in the Green's functions.

Evaluating the trace over spin, we notice a striking fact that an antisymmetric uniform $(q=0)$ component arises from the two noncommutative anomalous velocities proportional to $\boldsymbol{a}_{j} \times \boldsymbol{a}_{k}$ (see Appendix A). We have

$$
\pi_{i j k}(0)=-\epsilon_{i j k} c
$$

where

$$
c=\frac{4}{3} \lambda^{2} \frac{e}{V} \sum_{k} k \operatorname{Im}\left(f_{k}^{\mathrm{r}} h_{k}^{\mathrm{a}}\right)
$$

is a bulk chiral coefficient $(k \equiv|\boldsymbol{k}|)$. Here $f_{\boldsymbol{k}}^{\lambda}=\frac{1}{2} \sum_{\sigma= \pm} g_{\boldsymbol{k} \sigma}^{\lambda}$, $h_{\boldsymbol{k}}^{\lambda}=\frac{1}{2} \sum_{\sigma= \pm} \sigma g_{\boldsymbol{k} \sigma}^{\lambda}, g_{\boldsymbol{k} \sigma}^{\mathrm{r}} \equiv \frac{1}{-\epsilon_{k}-\sigma \gamma_{k}+\frac{i}{2 \tau}}$ is a spin-diagonalized Green's function, and $\gamma_{k} \equiv\left|\gamma_{k}\right|$.

The contributions to $\pi_{i j k}$ that are linear in $\boldsymbol{q}$ represent viscosity and pressure. They consist of symmetric and antisymmetric parts $\left(\pi_{i j k}^{(1)}=\pi_{i j k}^{(1) \mathrm{s}}+\pi_{i j k}^{(1) \mathrm{a}}\right)$,

$$
\pi_{i j k}^{(1) \mathrm{s}}=-i P q_{k} \delta_{i j}+i \eta_{\mathrm{s}}\left(q_{i} \delta_{j k}+q_{j} \delta_{i k}\right)
$$

and

$$
\pi_{i j k}^{(1) \mathrm{a}}=-i \eta_{\mathrm{a}}\left(q_{i} \delta_{j k}-q_{j} \delta_{i k}\right),
$$

respectively. The antisymmetric viscosity constant is (explicit expressions for $P$ and $\eta_{\mathrm{s}}$ are suppressed) (see Appendix A)

$$
\begin{aligned}
\eta_{\mathrm{a}}= & -\frac{\lambda^{2}}{3 m} \frac{e}{V} \operatorname{Im} \sum_{k} k^{2}\left[\frac{1}{\gamma_{k}} f_{k}^{\mathrm{r}} h_{k}^{\mathrm{a}}+f_{k}^{\mathrm{r}} f_{k}^{\mathrm{a}(2)}-h_{k}^{\mathrm{r}} h_{k}^{\mathrm{a}(2)}\right. \\
& \left.+\frac{m \lambda}{k}\left(f_{k}^{\mathrm{r}} h_{k}^{\mathrm{a}(2)}-h_{k}^{\mathrm{r}} f_{k}^{\mathrm{a}(2)}\right)\right],
\end{aligned}
$$

where $f_{\boldsymbol{k}}^{\lambda(2)} \equiv \frac{1}{2} \sum_{\sigma= \pm}\left(g_{k \sigma}^{\lambda}\right)^{2}$ and similarly for $h_{\boldsymbol{k}}^{\lambda(2)}$. Summing over $\boldsymbol{k}$, the bulk chiral coefficient is

$$
c=\frac{\pi}{3} \frac{e \lambda^{2}}{a^{3}} \sum_{\sigma} \frac{v_{\sigma} \gamma_{\sigma} k_{\sigma}}{\left(\gamma_{\sigma}\right)^{2}+\frac{1}{4 \tau^{2}}},
$$


where $v_{\sigma}$ and $k_{\sigma}$ are the spin-resolved electron density of states per site and the wave vector, respectively, $\gamma_{\sigma} \equiv \gamma_{k_{\sigma}}$. In the clean limit, $\gamma_{\sigma} \tau \gg 1, c \simeq \frac{\pi}{3} \frac{e \lambda^{2}}{a^{3}} \sum_{\sigma} \frac{v_{\sigma} k_{\sigma}}{\gamma_{\sigma}}$, while $c \simeq$ $\frac{4 \pi}{3} \frac{e \lambda^{2}}{a^{3}} \sum_{\sigma} v_{\sigma} \gamma_{\sigma} k_{\sigma} \tau^{2}$ in the disordered limit $\gamma_{\sigma} \tau \ll 1$. As shown in Appendix $\mathrm{D}$, the rotational viscosity is similarly evaluated to obtain $\eta_{\mathrm{a}} / c \simeq\left(k_{F}\right)^{-1}$, where $k_{F}$ is the Fermi energy, for both clean and dirty limits.

Including the force due to the external field and relaxation [10] (although they cancel each other in the steady state), the coarse-grained equation for $\dot{\boldsymbol{p}}$ in a chiral system is

$$
\begin{aligned}
\dot{\boldsymbol{p}}= & -c(\boldsymbol{\nabla} \times \boldsymbol{E})-\left(P-\eta_{\mathrm{s}}\right) \nabla(\nabla \cdot \boldsymbol{E})+\eta_{\mathrm{s}} \nabla^{2} \boldsymbol{E} \\
& +\eta_{\mathrm{a}}[\nabla \times(\nabla \times \boldsymbol{E})]+e n \boldsymbol{E}+\boldsymbol{f}_{\text {rel }} .
\end{aligned}
$$

The uniform component of the relaxation force $f_{\text {rel }}$ as a function of applied field turns out to be $f_{\text {rel }}=-e n \boldsymbol{E}$ (see Appendix E), and it therefore cancels the driving force due to applied field in the static case.

A hydrodynamic equation describing long-range behaviors compared to the mean free path is obtained by using a constitutive equation in the Ohmic regime, $j_{i}=(\sigma)_{i j} E_{j}$, where $\sigma$ is local conductivity tensor. It is obtained microscopically on the same footing as Eq. (12), with a result (up to the first order of q) $j_{i}(\boldsymbol{q})=\sigma_{\mathrm{e}} \delta_{i j} E_{j}+i \sigma_{0}^{\mathrm{a}} \epsilon_{i j k} q_{j} E_{k}$ (see Appendix B), i.e., $\boldsymbol{E}=$ $\frac{1}{\sigma_{\mathrm{e}}} \boldsymbol{j}-\frac{\sigma_{0}^{\mathrm{a}}}{\sigma_{\mathrm{e}}^{2}} \boldsymbol{\nabla} \times \boldsymbol{j}$. Neglecting third-order spatial derivatives, we finally obtain a standard hydrodynamic equation relating the force and current density,

$$
\begin{aligned}
\dot{\boldsymbol{p}}= & -\tilde{c}(\boldsymbol{\nabla} \times \boldsymbol{j})-\left(\tilde{P}-\tilde{\eta}_{\mathrm{s}}\right) \nabla(\nabla \cdot \boldsymbol{j})+\tilde{\eta}_{\mathrm{s}} \nabla^{2} \boldsymbol{j} \\
& +\tilde{\eta}_{\mathrm{a}}[\boldsymbol{\nabla} \times(\boldsymbol{\nabla} \times \boldsymbol{j})],
\end{aligned}
$$

with $\tilde{c} \equiv c / \sigma_{\mathrm{e}}, \tilde{P} \equiv P / \sigma_{\mathrm{e}}, \tilde{\eta}_{\mathrm{s}} \equiv \eta_{\mathrm{s}} / \sigma_{\mathrm{e}}$, and $\tilde{\eta}_{\mathrm{a}} \equiv \frac{1}{\sigma_{\mathrm{e}}}\left[\frac{\eta_{\mathrm{a}}}{\sigma_{\mathrm{e}}}+c \frac{\sigma_{0}^{\mathrm{a}}}{\sigma_{\mathrm{e}}}\right]$. The off-diagonal term of the conductivity tensor $\left(\sigma_{0}^{\mathrm{d}}\right)$ affects only the antisymmetric viscosity coefficient $\tilde{\eta}_{\mathrm{a}}$, but the correction is smaller than the diagonal contribution by the order of $\left(\lambda k_{\mathrm{F}} / \epsilon_{\mathrm{F}}\right)^{3}$. We clearly see that vorticity of the flow, $\boldsymbol{\nabla} \times \boldsymbol{j}$, plays essential roles of generating a global force $(c$ term) and rotational viscosity $\left(\eta_{\mathrm{a}}\right.$ term). The equation for chiral fluid, obtained microscopically for the Weyl electron system, applies to general chiral matter. In fact, the same equation has been argued on the phenomenological basis in the context of chiral active matter for describing biological phenomena $[3,4]$. The global chiral term $c$ was argued also in noncentrosymmetric metals and was shown based on a semiclassical argument [15].

\section{CHIRAL ANGULAR MOMENTUM GENERATION}

Chiral effects in Eq. (13) arise from rotational flow or vorticity, $\boldsymbol{\nabla} \times \boldsymbol{E}$ and $\boldsymbol{\nabla} \times \boldsymbol{j}$. The term $\eta_{\mathrm{a}}$ represents the rotational viscosity, while the bulk chiral coefficient, $c$, represents a global angular momentum generation. The roles of chiral terms become clear focusing on the total angular momentum of the system, $\boldsymbol{L}=\int d^{3} r(\boldsymbol{r} \times \boldsymbol{p})$. Here $\boldsymbol{r}$ in the present fluid description is a coordinate (and not a dynamic variable), and the spatial integral is over the system with a finite volume, $V$. The torque (time derivative of $\boldsymbol{L}$ ) on the system is $\dot{L}_{i}=$ $\epsilon_{i j k} \int d^{3} r r_{j} \nabla_{l} \pi_{k l}$. As the current density and thus the field $\boldsymbol{E}$ and $\pi_{k l}$ vanish at the surface of the system, we use the integral

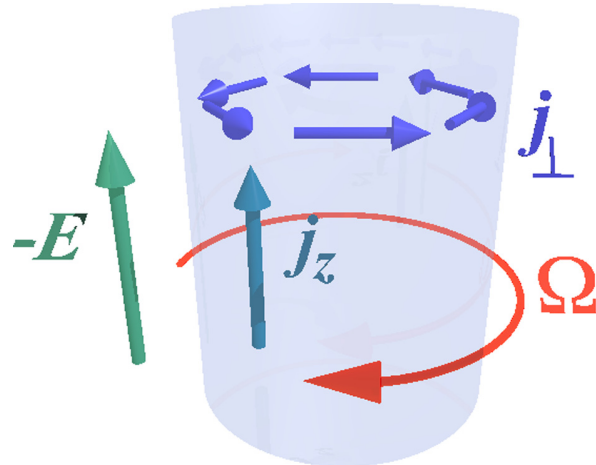

FIG. 1. Schematic figure of chiral angular momentum generation in a cylinder. An applied electric $\boldsymbol{E}$ field along the cylinder direction (chosen as the $z$ axis) induces a global rotation angular frequency $\Omega$ due to the chiral effect.

by parts to obtain

$$
\dot{L}_{i}=\epsilon_{i j k} \int d^{3} r \pi_{j k}^{\mathrm{a}}=\int d^{3} r\left[-\tilde{c} j_{i}+\tilde{\eta}_{\mathrm{a}}(\nabla \times j)_{i}\right],
$$

i.e., only the antisymmetric part of the momentum flux density, $\pi_{j k}^{\mathrm{a}}$, contributes to the total torque. Considering the $z$ component of the angular momentum when a uniform field is applied along the $z$ axis, $j_{z}$ for the $c$-term is written as the applied field, $E_{z}$, while the rotational contribution $(\nabla \times j)_{z}$ arises as a result of induced current $\boldsymbol{j}_{\perp}$ in the plane perpendicular to the applied field. The equation representing chiral angular momentum generation due to a driving field is therefore

$$
\dot{L}_{z}=\int d^{3} r\left[-c E_{z}+\tilde{\eta}_{\mathrm{a}}\left(\nabla \times \boldsymbol{j}_{\perp}\right)_{z}\right] .
$$

This equation applies to nonelectron cases if the field $\boldsymbol{E}$ is to be replaced by a corresponding driving force. The steady state is the one when the bulk chiral torque ( $c$ term) is balanced by the rotational viscosity.

Let us consider a cylinder along the $z$ axis with length $l_{z}$ and radius $R$ (Fig. 1). When an electric field is applied along the cylinder, an in-plane current perpendicular to the field is induced due to a chiral effect represented by antisymmetric components of the conductivity tensor (see Appendix B). Due to the rotational viscosity, the system reaches a steady state when the rotation is uniform with a constant angular frequency $\Omega$ determined by Eq. (15). In this steady state, the local current density in the $x y$-plane at $\boldsymbol{r}=(x, y)$ is $\boldsymbol{j}_{\perp}=$ $e n \Omega(\hat{z} \times \boldsymbol{r})$ ( $n$ is the electron density), and the local vortex density is uniform, $\left(\nabla \times \boldsymbol{j}_{\perp}\right)_{z}=2 e n \Omega$. The steady condition

$$
c E_{z}=\frac{\eta_{\mathrm{a}}}{\sigma_{\mathrm{e}}}\left(\nabla \times \boldsymbol{j}_{\perp}\right)_{z}
$$

is therefore satisfied locally at the angular frequency of

$$
\Omega=\frac{e E \tau}{m} \frac{c}{\eta_{\mathrm{a}}}=\frac{e E}{2} \frac{\ell}{\hbar},
$$

where $\ell=k_{F} \tau / m$ is the elastic electron mean free path, and we used $\sigma_{\mathrm{e}}=e^{2} n \tau / m$ and $c / \eta_{\mathrm{a}} \sim k_{F}$ in the last equality. The steady-state angular momentum of the electrons is $L_{e}=I_{e} \Omega$, where $I_{e}=\frac{m}{2} N R^{2}$ is the moment of inertia of the electrons, 
$N \equiv V / a^{3}$ being the number of lattice sites ( $a$ is the lattice constant). The electron angular momentum per site is thus

$$
\frac{L_{e}}{N}=e E \frac{m}{4 \hbar} \ell R^{2} .
$$

A significant feature here is the fact that it is proportional to the area of the cylinder. This is because the present chiral mechanism, Eq. (17), induces local vorticity, which is proportional to the angular frequency of the entire rotation. The total angular momentum is thus proportional to the area as a result of $I_{e} \propto R^{2}$. Equation (18) would, however, break down for large systems of $R \gg \ell_{L}$, where $\ell_{L}$ is a relaxation length of angular momentum.

The enhancement in large systems does not apply to the spin density induced by the electric field (Edelstein effect). The response function for the induced spin, discussed on the same footing as the orbital angular momentum, is $s_{j k}(\boldsymbol{q})=$ $\frac{e}{V} \sum_{k} \operatorname{tr}\left[\sigma_{j} G_{k}^{\mathrm{r}} \hat{v}_{k} G_{k+q}^{\mathrm{a}}\right]$ (see Appendix C). A uniform component exists, which is diagonal; $s_{j k}=\delta_{j k} \kappa_{s}$, where

$$
\kappa_{s}=2 \frac{e}{V} \sum_{k} \operatorname{Re}\left[\frac{2 k}{3 m} f_{k}^{\mathrm{r}} h_{k}^{\mathrm{a}}+\lambda\left(f_{k}^{\mathrm{r}} f_{k}^{\mathrm{a}}-\frac{1}{3} h_{k}^{\mathrm{r}} h_{k}^{\mathrm{a}}\right)\right] .
$$

The induced spin density is (with $\hbar$ recovered)

$$
s=\kappa_{s} E \simeq \frac{e E}{\hbar} \frac{\tau}{k_{F}} .
$$

The induced spin density is determined simply by microscopic material parameters. The ratio of the chirality-induced orbital and spin angular momenta is significantly large for large systems; $\frac{L_{e}}{N} / s \simeq(R / a)^{2}$. Although both are angular momenta, the efficiency for electric generation is an order of magnitude larger for the orbital one compared to the spin because of Eq. (18). This large pumped angular momentum by electron injection, combined with spin-orbit interaction, may account for the large spin polarization of the electrons transmitted through chiral materials (spin selectivity) [25].

Let us look into the numbers. If we apply a voltage of $1 \mathrm{~V}$ along a cylinder of $l_{z}=1 \mu \mathrm{m}, r=100 \mathrm{~nm}, e E a=$ $3.2 \times 10^{-23} \mathrm{~J}$ for $a=2 \AA$. The angular momentum in units of $\hbar$ per site is $\frac{L_{e}}{\hbar N} \simeq \frac{e E a}{\epsilon_{F}}\left(k_{F} R\right)^{2} \frac{\ell}{a}=0.5 \times 10^{4}$ for $\ell / a=100$ and $k_{F} \sim a^{-1}$ and $\epsilon_{F}=1 \mathrm{eV}$, while the induced spin $s$ is $4 \times 10^{-6}$ times smaller.

When the cylinder is free to rotate, the angular momentum of the electrons is distributed to the rotation of the whole system. The rotation angular frequency in this case is $L_{\text {tot }}=$ $\frac{m}{N_{\mathrm{a}} m_{\mathrm{p}}} L_{\mathrm{e}}$, where $m_{\mathrm{p}}$ and $N_{\mathrm{a}}$ are the proton mass and atomic number. Considering the case of a $\mathrm{C}$ atom $\left(N_{\mathrm{a}}=12\right)$, the suppression factor is $\frac{m}{N_{\mathrm{a}} m_{\mathrm{p}}}=4.5 \times 10^{-5}$. If the orbital angular momentum estimated above is fully transferred to a mechanical rotation, it corresponds to the angular frequency of the total system of $\Omega_{\text {tot }}=\frac{m}{N_{\mathrm{a}} m_{\mathrm{p}}} \Omega \sim \frac{m}{N_{\mathrm{a}} m_{\mathrm{p}}} e E a \frac{\ell}{\hbar a}=7 \times 10^{8} \mathrm{~Hz}$.

Equation (17) indicates that chiral systems work as rotation sensors detected electrically. The voltage at a steady angular frequency $\Omega$ is $V_{E}=E l_{z}=\frac{2 \hbar}{e} \frac{l_{z}}{\ell} \Omega$, which in the above case is $V_{E}=0.7 \times 10^{-13} \times \Omega[\mathrm{Hz}] \mathrm{V}$. For a device of $l_{z}=1 \mathrm{~mm}$, the magnitude is of the order of $0.1 \mu \mathrm{V}$ for $\Omega=1 \mathrm{kHz}$ and would be detectable.

\section{DISCUSSION}

As our results [Eq. (11)] indicate, the bulk chiral coefficient and rotational viscosity have different behaviors in the clean $\left(\gamma_{\sigma} \tau \gg 1\right)$ and dirty $\left(\gamma_{\sigma} \tau \ll 1\right)$ limits. Experimental control of $\gamma_{\sigma}$ by tuning the Fermi level would be of interest.

The angular momentum generation effect suggests the existence of a chiral edge current. In fact, by calculating the electric conductivity tensor, we find a chiral contribution, antisymmetric and linear in $q, \sigma_{j k}^{\mathrm{ch}}=\sigma_{\mathrm{a}} i \epsilon_{j k l} q_{l}$, where $\sigma_{\mathrm{a}}$ is a constant (see Appendix B). This contribution represents a chiral current circulating in the plane perpendicular to the applied field at the edge of the system, where the $\nabla \times j$ is finite. This chiral current, argued in Ref. [15], is interpreted as another aspect of bulk chiral angular momentum generation.

Electric generation of orbital angular momentum in a chiral crystal was theoretically pointed out in Ref. [26] (called the orbital Edelstein effect). A realization in honeycomb-lattice layers was argued. The approach of those authors is based on a semiclassical Berry phase formula, and thus it is an equilibrium contribution. Their result of induced magnetization was proportional to the elastic lifetime $\tau$, which agrees with the present dynamic approach, Eq. (18). A Berry phase interpretation of the chiral coefficient $c$ was argued in Ref. [15].

Equation (15) indicates that the rotational viscosity contributes to the diamagnetic constant. In fact, using $\nabla \times \boldsymbol{E}=$ $-\dot{\boldsymbol{B}}$ we obtain a standard diamagnetism relation, $\boldsymbol{L} / V=$ $-\eta_{\mathrm{a}} \boldsymbol{B}$, when a magnetic field $\boldsymbol{B}$ is applied without $\boldsymbol{E}$. Note that the diamagnetic effect represented by the viscosity is only the dynamic contribution, as it was derived by evaluating $\dot{\boldsymbol{L}}$.

\section{A. Electromagnetic viewpoint}

From an electromagnetic viewpoint, the bulk chiral contribution, $\dot{\boldsymbol{L}} \propto c \boldsymbol{E}$ [Eq. (15)], is a cross-correlational diamagnetic response to the electric field. Mathematically, the effect arises from a noncommutative off-diagonal contribution in the response function (see Appendix A). Obviously, inversion symmetry breaking (chiral nature) is essential; for example, $c$ vanishes for the case of the Rashba interaction, $\boldsymbol{\gamma}_{k}=\boldsymbol{\alpha} \times \boldsymbol{k}$ ( $\alpha$ is the Rashba vector). The existence of spin or pseudospin (sublattice) in addition to the parity symmetry breaking is therefore essential for the bulk chiral effect. The requirement is common to chiral and topological effects, suggesting a close relation between the two. This point is further discussed from the viewpoint of electromagnetic response.

\section{B. Chiral versus topological effects}

The induced angular momentum $\boldsymbol{L}$ of charge leads to a magnetization, $\boldsymbol{M}=\frac{e}{2 m} \boldsymbol{L}$. Our result, Eq. (18), therefore indicates that a coupling between $\boldsymbol{E}$ and $\dot{\boldsymbol{B}}$ is induced by chirality. This is consistent with a recent finding [27] that electromagnetism in chiral material contains an effective coupling proportional to the chirality order parameter, $C \equiv \boldsymbol{E}$. $(\nabla \times \boldsymbol{E})=-\boldsymbol{E} \cdot \dot{\boldsymbol{B}}$. This is natural as the coupling $\boldsymbol{E} \cdot \dot{\boldsymbol{B}}$ is chiral in the sense that it breaks parity invariance while maintaining time-reversal invariance [28]. From this electromagnetism viewpoint, our chiral angular momentum effect is closely related to topological effects. In fact, the effective Hamiltonian for the electromagnetism in topological 
insulators is proportional to $\boldsymbol{E} \cdot \boldsymbol{B}$, resulting in a direct coupling $\boldsymbol{M} \propto \boldsymbol{E}$ [29] or $\boldsymbol{L} \propto \boldsymbol{E}$. Therefore, our dynamic bulk chiral effect is a nonequilibrium counterpart of topological effects, inducing $\dot{\boldsymbol{L}}$ instead of $\boldsymbol{L}$ itself. Moreover, the dynamic chiral effect leads to a steady state with a finite angular momentum [Eq. (18)], i.e., it has qualitatively the same effect as the topological one. The field-induced orbital magnetization observed in experiments is thus written as

$$
\boldsymbol{M}=c_{\mathrm{top}} \boldsymbol{E}+c_{\mathrm{ch}} \boldsymbol{E},
$$

where $c_{\text {top }}$ is a coefficient for the topological effect, and $c_{\text {ch }} \propto$ $c$ represents the present dynamic chiral effect. Being topological, $c_{\text {top }}$ is a universal constant, while $c_{\text {ch }}$ arising as a result of relaxation due to viscosity is of the order of $c_{\mathrm{ch}} / c_{\text {top }} \simeq$ $\tau_{L} \varepsilon$, where $\tau_{L}$ and $\varepsilon$ represent the relaxation time for the angular momentum and the typical energy scale, respectively. In the present simple model, $\varepsilon \sim \epsilon_{F}$ and $\tau_{L} \propto \tau$. For weak angular momentum relaxation, the ratio is much larger than unity, suggesting that the topological contribution might be smeared out.

\section{CONCLUSION}

To conclude, we have constructed a hydrodynamic theory to describe chiral electron systems by a straightforward calculation on a quantum field-theoretical basis. Deriving the equation of motion for the orbital angular momentum, we discussed a bulk chiral angular momentum generation effect when an electric field is applied. The orbital angular momentum is orders of magnitude larger than the spin angular momentum. The hydrodynamic equation itself applies generally for any chiral fluids if the electric field is replaced by the corresponding driving field. In the present formalism, the effect of magnetic field is taken into account systematically by an expansion in terms of $q$ at the static limit $\left(\omega_{0} \rightarrow 0\right)$.

\section{ACKNOWLEDGMENTS}

The authors thank S. Seki, Y. Fuseya, and J. Kishine for valuable discussion, and A. Shitade for useful comments on the validity of the model. This study was supported by a Grant-in-Aid for Scientific Research (B) (No. 17H02929) from the Japan Society for the Promotion of Science.

\section{APPENDIX A: MOMENTUM FLUX TENSOR}

The Hamiltonian we consider is

$$
H=\sum_{k} \hat{c}_{k}^{\dagger}\left(\epsilon_{k}+\gamma_{k} \cdot \sigma\right) \hat{c}_{k},
$$

where $\hat{c}$ and $\hat{c}^{\dagger}$ are electron field operators with two spin components, and $\epsilon_{\boldsymbol{k}} \equiv \frac{k^{2}}{2 m}-\epsilon_{F}$ is the free-electron energy $\left(\epsilon_{F}\right.$ is the Fermi energy, $m$ is the electron mass). A vector $\boldsymbol{\gamma}_{\boldsymbol{k}}$ represents the spin-orbit interaction, $\sigma$ being a vector of Pauli matrices. We consider the Weyl type,

$$
\gamma_{k}=\lambda \boldsymbol{k},
$$

with $\lambda$ a coupling constant. The velocity operator is

$$
\hat{v}_{i}=\frac{k_{i}}{m}+a_{i}^{\alpha} \sigma_{\alpha},
$$

where

$$
a_{i}^{\alpha}=\frac{d \gamma_{k}^{\alpha}}{d k_{i}}=\lambda \delta_{i \alpha}
$$

is the anomalous velocity due to the spin-orbit interaction.

The explicit expression for the response function for the momentum flux tensor is

$$
\begin{aligned}
\pi_{i j k}(\boldsymbol{q})= & \sum_{\boldsymbol{k}} \operatorname{tr}\left[k_{i}\left(\frac{k_{j}}{m}+\boldsymbol{a}_{j} \cdot \boldsymbol{\sigma}\right)\left(f_{\boldsymbol{k}-\frac{q}{2}}^{\mathrm{r}}+h_{\boldsymbol{k}-\frac{q}{2}}^{\mathrm{r}} \hat{\gamma}_{\boldsymbol{k}-\frac{q}{2}} \cdot \boldsymbol{\sigma}\right)\right. \\
& \left.\times\left(\frac{k_{k}}{m}+\boldsymbol{a}_{k} \cdot \boldsymbol{\sigma}\right)\left(f_{\boldsymbol{k}+\frac{q}{2}}^{\mathrm{a}}+h_{k+\frac{q}{2}}^{\mathrm{a}} \hat{\gamma}_{\boldsymbol{k}+\frac{q}{2}} \cdot \boldsymbol{\sigma}\right)\right],
\end{aligned}
$$

where $(\lambda=r, a)$

$$
\begin{aligned}
f_{k}^{\lambda} & \equiv \frac{1}{2} \sum_{\sigma= \pm} g_{k \sigma}^{\lambda}, \\
h_{k}^{\lambda} & \equiv \frac{1}{2} \sum_{\sigma= \pm} \sigma g_{k \sigma}^{\lambda},
\end{aligned}
$$

with

$$
g_{k \sigma}^{\mathrm{r}} \equiv \frac{1}{-\epsilon_{k}-\sigma \gamma_{k}+\frac{i}{2 \tau}}
$$

being the diagonalized Green's function, $g_{\boldsymbol{k} \sigma}^{\mathrm{a}}=\left(g_{\boldsymbol{k} \sigma}^{\mathrm{r}}\right)^{*}$.

Evaluating the trace in spin, it reduces to

$$
\begin{aligned}
\pi_{i j k}(\boldsymbol{q}) & \\
= & 2 \sum_{\boldsymbol{k}}\left[k_{i} \frac{k_{j} k_{k}}{m^{2}}\left[f_{\boldsymbol{k}-\frac{q}{2}}^{\mathrm{r}} f_{\boldsymbol{k}+\frac{q}{2}}^{\mathrm{a}}+\left(\hat{\boldsymbol{\gamma}}_{\boldsymbol{k}-\frac{q}{2}} \cdot \hat{\boldsymbol{\gamma}}_{\boldsymbol{k}+\frac{q}{2}}\right) h_{\boldsymbol{k}+\frac{q}{2}}^{\mathrm{r}} h_{\boldsymbol{k}-\frac{q}{2}}^{\mathrm{a}}\right]\right. \\
& +\frac{k_{i}}{m}\left(k_{j} \boldsymbol{a}_{k}+k_{k} \boldsymbol{a}_{j}\right) \cdot\left(f_{\boldsymbol{k}-\frac{q}{2}}^{\mathrm{r}} h_{\boldsymbol{k}+\frac{q}{2}}^{\mathrm{a}} \hat{\gamma}_{\boldsymbol{k}+\frac{q}{2}}+f_{\boldsymbol{k}+\frac{q}{2}}^{\mathrm{a}} h_{\boldsymbol{k}-\frac{q}{2}}^{\mathrm{r}} \hat{\boldsymbol{\gamma}}_{\boldsymbol{k}-\frac{q}{2}}\right) \\
& -i \frac{k_{i}}{m}\left(k_{j} \boldsymbol{a}_{k}-k_{k} \boldsymbol{a}_{j}\right) \cdot\left(\hat{\boldsymbol{\gamma}}_{\boldsymbol{k}-\frac{q}{2}} \times \hat{\boldsymbol{\gamma}}_{\boldsymbol{k}+\frac{q}{2}}\right) h_{\boldsymbol{k}-\frac{q}{2}}^{\mathrm{r}} h_{\boldsymbol{k}+\frac{q}{2}}^{\mathrm{a}} \\
& +k_{i}\left(\boldsymbol{a}_{j} \cdot \boldsymbol{a}_{k}\right)\left[f_{\boldsymbol{k}-\frac{q}{2}}^{\mathrm{r}} f_{\boldsymbol{k}+\frac{q}{2}}^{\mathrm{a}}-\left(\hat{\boldsymbol{\gamma}}_{\boldsymbol{k}-\frac{q}{2}} \cdot \hat{\boldsymbol{\gamma}}_{\boldsymbol{k}+\frac{q}{2}}\right) h_{\boldsymbol{k}-\frac{q}{2}}^{\mathrm{r}} h_{\boldsymbol{k}+\frac{q}{2}}^{\mathrm{a}}\right] \\
& +i k_{i}\left(\boldsymbol{a}_{j} \times \boldsymbol{a}_{k}\right) \cdot\left(\hat{\boldsymbol{\gamma}}_{\boldsymbol{k}+\frac{q}{2}} f_{\boldsymbol{k}-\frac{q}{2}}^{\mathrm{r}} h_{\boldsymbol{k}+\frac{q}{2}}^{\mathrm{a}}-\hat{\boldsymbol{\gamma}}_{\boldsymbol{k}-\frac{q}{2}} f_{\boldsymbol{k}+\frac{q}{2}}^{\mathrm{a}} h_{\boldsymbol{k}-\frac{q}{2}}^{\mathrm{r}}\right) \\
& \left.+k_{i}\left(a_{j}^{\alpha} a_{k}^{\beta}+a_{k}^{\alpha} a_{j}^{\beta}\right) \hat{\boldsymbol{\gamma}}_{\boldsymbol{k}-\frac{q}{2}}^{\alpha} \hat{\boldsymbol{\gamma}}_{\boldsymbol{k}+\frac{q}{2}}^{\beta} h_{\boldsymbol{k}-\frac{q}{2}}^{\mathrm{r}} h_{\boldsymbol{k}+\frac{q}{2}}^{\mathrm{a}}\right] .
\end{aligned}
$$

For a linear spin-orbit interaction that we consider, $a_{j}^{\alpha}$ and $f_{k}$ are even in $\boldsymbol{k}$ while $h_{\boldsymbol{k}}$ is odd. The uniform $(q=0)$ component of the response function is therefore asymmetric with respect to the directions $j$ and $k$ as

$$
\pi_{i j k}(0)=-4 \sum_{\boldsymbol{k}} k_{i}\left(\boldsymbol{a}_{j} \times \boldsymbol{a}_{k}\right) \cdot \hat{\boldsymbol{\gamma}}_{\boldsymbol{k}} \operatorname{Im}\left(f_{\boldsymbol{k}}^{\mathrm{r}} h_{\boldsymbol{k}}^{\mathrm{a}}\right) \equiv \epsilon_{j k l} c_{i l} .
$$

\section{Weyl case}

For the Weyl type, we have

$$
a_{j}^{\alpha}=\lambda \delta_{i \alpha}, \quad \hat{\gamma}_{k}=\hat{k}
$$


and $\left(\boldsymbol{a}_{j} \times \boldsymbol{a}_{k}\right) \cdot \boldsymbol{\gamma}_{\boldsymbol{k}}=\lambda^{2} \epsilon_{j k l} \hat{\boldsymbol{k}}_{l}$. We thus have

$$
\pi_{i j k}(0)=-\epsilon_{i j k} c
$$

where

$$
c=-\frac{4}{3} \lambda^{2} \sum_{k} k \operatorname{Im}\left(f_{k}^{\mathrm{r}} h_{k}^{\mathrm{a}}\right)=\frac{8}{3} \lambda^{2} \sum_{k} k \operatorname{Im}\left[g_{k-}^{\mathrm{r}} g_{+}^{\mathrm{a}}\right]
$$

is the bulk chirality coefficient of the Weyl system.

\section{Rashba case}

For the Rashba spin-orbit interaction with the Rashba vector $\boldsymbol{\alpha}$, we have

$$
a_{j}^{\alpha}=\alpha_{\beta} \epsilon_{j \alpha \beta}, \quad \hat{\gamma}_{\boldsymbol{k}}=(\boldsymbol{\alpha} \times \boldsymbol{k}) / \gamma_{\boldsymbol{k}} .
$$

Thus $\left(\boldsymbol{a}_{j} \times \boldsymbol{a}_{k}\right) \cdot \boldsymbol{\gamma}_{\boldsymbol{k}}=\epsilon_{j \alpha \beta} \epsilon_{k \gamma \delta} \epsilon_{\beta \delta l} \alpha_{\alpha} \alpha_{\gamma}(\boldsymbol{\alpha} \times \boldsymbol{k})_{l}=\left(\delta_{j \delta} \delta_{\alpha l}-\right.$ $\left.\delta_{j l} \delta_{\alpha \delta}\right) \epsilon_{k \gamma \delta} \alpha_{\alpha} \alpha_{\gamma}(\boldsymbol{\alpha} \times \boldsymbol{k})_{l}=0$, and the uniform component vanishes. The Dresselhaus interaction also leads to a vanishing bulk chiral coefficient.

$q$-linear terms of $\pi_{i j k}, \pi_{i j k}^{(1)}$ represent viscosity of the fluid. We expand $\pi_{i j k}(\boldsymbol{q})$ with respect to $\boldsymbol{q}$ to linear order using

$$
f_{k-\frac{q}{2}}^{\mathrm{r}} f_{k+\frac{q}{2}}^{\mathrm{a}}=f_{k}^{\mathrm{r}} f_{k}^{\mathrm{a}}+\frac{q_{l}}{2}\left(f_{k}^{\mathrm{r}} \stackrel{\leftrightarrow}{\partial}_{k_{l}} f_{k}^{\mathrm{a}}\right)+O\left(q^{2}\right)
$$

The linear order contribution is then

$$
\begin{aligned}
\pi_{i j k}^{(1)}(\boldsymbol{q})= & \sum_{\boldsymbol{k}} q_{l}\left[\frac{1}{m^{2}} k_{i} k_{j} k_{k}\left(f_{\boldsymbol{k}}^{\mathrm{r}} \stackrel{\leftrightarrow}{\partial}_{k_{l}} f_{\boldsymbol{k}}^{\mathrm{a}}+\hat{\gamma}_{\boldsymbol{k}}^{\alpha} h_{\boldsymbol{k}}^{\mathrm{r}} \stackrel{\leftrightarrow}{\partial}_{k_{l}} \hat{\gamma}_{\boldsymbol{k}}^{\alpha} h_{\boldsymbol{k}}^{\mathrm{a}}\right)+\frac{1}{m} k_{i}\left(k_{j} a_{k}^{\alpha}+k_{k} a_{j}^{\alpha}\right)\left(f_{\boldsymbol{k}}^{\mathrm{r}} \stackrel{\leftrightarrow}{\partial}_{k_{l}} \hat{\gamma}_{\boldsymbol{k}}^{\alpha} h_{\boldsymbol{k}}^{\mathrm{a}}-\text { c.c. }\right)\right. \\
& \left.+k_{i}\left(\boldsymbol{a}_{j} \cdot \boldsymbol{a}_{k}\right)\left(f_{\boldsymbol{k}}^{\mathrm{r}} \stackrel{\leftrightarrow}{\partial}_{k_{l}} f_{\boldsymbol{k}}^{\mathrm{a}}-\hat{\gamma}_{\boldsymbol{k}}^{\alpha} h_{\boldsymbol{k}}^{\mathrm{r}} \stackrel{\leftrightarrow}{\partial}_{k_{l}} \hat{\gamma}_{\boldsymbol{k}}^{\alpha} h_{\boldsymbol{k}}^{\mathrm{a}}\right)+k_{i}\left(a_{j}^{\alpha} a_{k}^{\beta}+a_{k}^{\alpha} a_{j}^{\beta}\right)\left(\hat{\gamma}_{\boldsymbol{k}}^{\alpha} h_{\boldsymbol{k}}^{\mathrm{r}} \stackrel{\leftrightarrow}{\partial}_{k_{l}} \hat{\gamma}_{\boldsymbol{k}}^{\beta} h_{\boldsymbol{k}}^{\mathrm{a}}\right)\right] .
\end{aligned}
$$

Derivatives are written using

$$
\partial_{k_{l}} \hat{\gamma}_{\boldsymbol{k}}^{\alpha}=\frac{1}{\gamma_{\boldsymbol{k}}}\left[\boldsymbol{a}_{l}-\hat{\gamma}_{\boldsymbol{k}}\left(\hat{\gamma}_{\boldsymbol{k}} \cdot \boldsymbol{a}_{l}\right)\right]_{\alpha}
$$

as

$$
\begin{aligned}
\partial_{k_{l}} f_{\boldsymbol{k}}^{\mathrm{a}} & =\frac{1}{2} \sum_{\sigma}\left(\frac{k_{l}}{m}+\sigma \hat{\boldsymbol{\gamma}}_{\boldsymbol{k}} \cdot \boldsymbol{a}_{l}\right)\left(g_{\boldsymbol{k} \sigma}^{\mathrm{a}}\right)^{2}=\frac{k_{l}}{m} f_{\boldsymbol{k}}^{\mathrm{a}(2)}+\left(\hat{\boldsymbol{\gamma}}_{\boldsymbol{k}} \cdot \boldsymbol{a}_{l}\right) h_{\boldsymbol{k}}^{\mathrm{a}(2)}, \\
\partial_{k_{l}}\left(\hat{\gamma}_{\boldsymbol{k}}^{\alpha} h_{\boldsymbol{k}}^{\mathrm{a}}\right) & =\left(\partial_{k_{l}} \hat{\gamma}_{\boldsymbol{k}}^{\alpha}\right) h_{\boldsymbol{k}}^{\mathrm{a}}+\hat{\gamma}_{\boldsymbol{k}}^{\alpha} \partial_{k_{l}} h_{\boldsymbol{k}}^{\mathrm{a}} \\
& =\frac{1}{\gamma_{\boldsymbol{k}}}\left[\boldsymbol{a}_{l}-\hat{\gamma}_{\boldsymbol{k}}\left(\hat{\gamma}_{\boldsymbol{k}} \cdot \boldsymbol{a}_{l}\right)\right]_{\alpha} h_{\boldsymbol{k}}^{\mathrm{a}}+\hat{\gamma}_{\boldsymbol{k}}^{\alpha}\left(\frac{k_{l}}{m} h_{\boldsymbol{k}}^{\mathrm{a}(2)}+\left(\hat{\boldsymbol{\gamma}}_{\boldsymbol{k}} \cdot \boldsymbol{a}_{l}\right) f_{\boldsymbol{k}}^{\mathrm{a}(2)}\right),
\end{aligned}
$$

where

$$
\begin{aligned}
f_{\boldsymbol{k}}^{\mathrm{a}(n)} & \equiv \frac{1}{2} \sum_{\sigma}\left(g_{\boldsymbol{k} \sigma}^{\mathrm{a}}\right)^{n}, \\
h_{\boldsymbol{k}}^{\mathrm{a}(n)} & \equiv \frac{1}{2} \sum_{\sigma} \sigma\left(g_{\boldsymbol{k} \sigma}^{\mathrm{a}}\right)^{n} .
\end{aligned}
$$

We have therefore

$$
\begin{aligned}
& f_{\boldsymbol{k}}^{\mathrm{r}} \stackrel{\leftrightarrow}{\partial}_{k_{l}} f_{\boldsymbol{k}}^{\mathrm{a}}=2 i \operatorname{Im}\left[\frac{k_{l}}{m} f_{\boldsymbol{k}}^{\mathrm{r}} f_{\boldsymbol{k}}^{\mathrm{a}(2)}+\left(\hat{\gamma}_{\boldsymbol{k}} \cdot \boldsymbol{a}_{l}\right) f_{\boldsymbol{k}}^{\mathrm{r}} h_{\boldsymbol{k}}^{\mathrm{a}(2)}\right]=2 i \frac{k_{l}}{m} \operatorname{Im}\left[f_{\boldsymbol{k}}^{\mathrm{r}} f_{\boldsymbol{k}}^{\mathrm{a}(2)}+\frac{m \lambda}{k} f_{\boldsymbol{k}}^{\mathrm{r}} h_{\boldsymbol{k}}^{\mathrm{a}(2)}\right], \\
& \hat{\gamma}_{\boldsymbol{k}}^{\alpha} h_{\boldsymbol{k}}^{\mathrm{r}} \stackrel{\leftrightarrow}{\partial}_{k_{l}} \hat{\gamma}_{\boldsymbol{k}}^{\alpha} h_{\boldsymbol{k}}^{\mathrm{a}}=2 i \operatorname{Im}\left[\frac{k_{l}}{m} h_{\boldsymbol{k}}^{\mathrm{r}} h_{\boldsymbol{k}}^{\mathrm{a}(2)}+\left(\hat{\gamma}_{\boldsymbol{k}} \cdot \boldsymbol{a}_{l}\right) h_{\boldsymbol{k}}^{\mathrm{r}} f_{\boldsymbol{k}}^{\mathrm{a}(2)}\right]=2 i \frac{k_{l}}{m} \operatorname{Im}\left[\bar{h}_{\boldsymbol{k}}^{\mathrm{r}} \bar{h}_{\boldsymbol{k}}^{\mathrm{a}(2)}+\frac{m \lambda}{k} \bar{h}_{\boldsymbol{k}}^{\mathrm{r}} f_{\boldsymbol{k}}^{\mathrm{a}(2)}\right], \\
& f_{\boldsymbol{k}}^{\mathrm{r}} \stackrel{\leftrightarrow}{\partial}_{k_{l}} \hat{\gamma}_{\boldsymbol{k}}^{\alpha} h_{\boldsymbol{k}}^{\mathrm{a}}=\frac{1}{\gamma_{\boldsymbol{k}}}\left[\boldsymbol{a}_{l}-\hat{\gamma}_{\boldsymbol{k}}\left(\hat{\boldsymbol{\gamma}}_{\boldsymbol{k}} \cdot \boldsymbol{a}_{l}\right)\right]_{\alpha} f_{\boldsymbol{k}}^{\mathrm{r}} h_{\boldsymbol{k}}^{\mathrm{a}}+\hat{\gamma}_{\boldsymbol{k}}^{\alpha}\left[\frac{k_{l}}{m}\left(f_{\boldsymbol{k}}^{\mathrm{r}} h_{\boldsymbol{k}}^{\mathrm{a}(2)}-f_{\boldsymbol{k}}^{\mathrm{r}(2)} h_{\boldsymbol{k}}^{\mathrm{a}}\right)+\left(\hat{\boldsymbol{\gamma}}_{\boldsymbol{k}} \cdot \boldsymbol{a}_{l}\right)\left(f_{\boldsymbol{k}}^{\mathrm{r}} f_{\boldsymbol{k}}^{\mathrm{a}(2)}-h_{\boldsymbol{k}}^{\mathrm{r}(2)} h_{\boldsymbol{k}}^{\mathrm{a}}\right)\right] \\
&=\frac{\lambda}{\gamma_{\boldsymbol{k}}}\left[\delta_{l \alpha}-\hat{k}_{\alpha} \hat{k}_{l}\right] f_{\boldsymbol{k}}^{\mathrm{r}} h_{\boldsymbol{k}}^{\mathrm{a}}+\frac{\hat{k}_{l} \hat{k}_{\alpha}}{m}\left[f_{\boldsymbol{k}}^{\mathrm{r}} h_{\boldsymbol{k}}^{\mathrm{a}(2)}-f_{\boldsymbol{k}}^{\mathrm{r}(2)} h_{\boldsymbol{k}}^{\mathrm{a}}+\frac{m \lambda}{k}\left(f_{\boldsymbol{k}}^{\mathrm{r}} f_{\boldsymbol{k}}^{\mathrm{a}(2)}-h_{\boldsymbol{k}}^{\mathrm{r}(2)} h_{\boldsymbol{k}}^{\mathrm{a}}\right)\right]
\end{aligned}
$$

for the Weyl case. The asymmetric contribution for the Weyl case is found to be

$$
\pi_{i j k}^{(1) \mathrm{a}}=i \frac{\lambda^{2}}{3 m}\left(\delta_{i k} q_{j}-\delta_{j k} q_{i}\right) \operatorname{Im} \sum_{k} k^{2}\left[\frac{1}{\gamma_{k}} f_{k}^{\mathrm{r}} h_{\boldsymbol{k}}^{\mathrm{a}}+\left(f_{\boldsymbol{k}}^{\mathrm{r}} f_{\boldsymbol{k}}^{\mathrm{a}(2)}-h_{k}^{\mathrm{r}} h_{k}^{\mathrm{a}(2)}+\frac{m \lambda}{k}\left(f_{\boldsymbol{k}}^{\mathrm{r}} h_{\boldsymbol{k}}^{\mathrm{a}(2)}-h_{k}^{\mathrm{r}} f_{\boldsymbol{k}}^{\mathrm{a}(2)}\right)\right)\right] .
$$

In the case of the Rashba interaction, the antisymmetric component of viscosity is finite due to the anisotropic propagation, resulting in $\pi_{i j}^{(1) a}=\pi_{i j k}^{(1) a} E_{k} \propto i\left(q_{i} \alpha_{j}-q_{j} \alpha_{i}\right)(\boldsymbol{\alpha} \cdot \boldsymbol{E})$ and $i\left(\alpha_{i} E_{j}-\alpha_{j} E_{i}\right)(\boldsymbol{q} \cdot \boldsymbol{\alpha})$. 
Antisymmetric viscosity arises in the presence of anomalous velocity $\left(\boldsymbol{a}_{i} \cdot \boldsymbol{\sigma}\right)$, as it requires noncollinear alignment of momentum and velocity.

\section{APPENDIX B: CONDUCTIVITY}

The correlation function $\sigma$ for the conductivity is

$$
\begin{aligned}
\sigma_{j k}(\boldsymbol{q})= & 2 \sum_{\boldsymbol{k}}\left[\frac{k_{j} k_{k}}{m^{2}}\left[f_{\boldsymbol{k}-\frac{q}{2}}^{\mathrm{r}} f_{\boldsymbol{k}+\frac{q}{2}}^{\mathrm{a}}+\left(\hat{\gamma}_{\boldsymbol{k}-\frac{q}{2}} \cdot \hat{\boldsymbol{\gamma}}_{\boldsymbol{k}+\frac{q}{2}}\right) h_{\boldsymbol{k}+\frac{q}{2}}^{\mathrm{r}} h_{\boldsymbol{k}-\frac{q}{2}}^{\mathrm{a}}\right]\right. \\
& +\frac{1}{m}\left(k_{j} \boldsymbol{a}_{k}+k_{k} \boldsymbol{a}_{j}\right) \cdot\left(\hat{\gamma}_{\boldsymbol{k}+\frac{q}{2}} f_{\boldsymbol{k}-\frac{q}{2}}^{\mathrm{r}} h_{\boldsymbol{k}+\frac{q}{2}}^{\mathrm{a}}+\hat{\boldsymbol{\gamma}}_{\boldsymbol{k}-\frac{q}{2}} f_{\boldsymbol{k}+\frac{q}{2}}^{\mathrm{a}} h_{\boldsymbol{k}-\frac{q}{2}}^{\mathrm{r}}\right)-i \frac{1}{m}\left(k_{j} \boldsymbol{a}_{k}-k_{k} \boldsymbol{a}_{j}\right) \cdot\left(\hat{\boldsymbol{\gamma}}_{\boldsymbol{k}-\frac{q}{2}} \times \hat{\boldsymbol{\gamma}}_{\boldsymbol{k}+\frac{q}{2}}\right) h_{\boldsymbol{k}-\frac{q}{2}}^{\mathrm{r}} h_{\boldsymbol{k}+\frac{q}{2}}^{\mathrm{a}} \\
& +\left(\boldsymbol{a}_{j} \cdot \boldsymbol{a}_{k}\right)\left[f_{\boldsymbol{k}-\frac{q}{2}}^{\mathrm{r}} f_{\boldsymbol{k}+\frac{q}{2}}^{\mathrm{a}}-\left(\hat{\gamma}_{\boldsymbol{k}-\frac{q}{2}} \cdot \hat{\boldsymbol{\gamma}}_{\boldsymbol{k}+\frac{q}{2}}\right) h_{\boldsymbol{k}-\frac{q}{2}}^{\mathrm{r}} h_{\boldsymbol{k}+\frac{q}{2}}^{\mathrm{a}}\right]+i\left(\boldsymbol{a}_{j} \times \boldsymbol{a}_{k}\right) \cdot\left(\hat{\boldsymbol{\gamma}}_{\boldsymbol{k}+\frac{q}{2}} f_{\boldsymbol{k}-\frac{q}{2}}^{\mathrm{r}} h_{\boldsymbol{k}+\frac{q}{2}}^{\mathrm{a}}-\hat{\boldsymbol{\gamma}}_{\boldsymbol{k}-\frac{q}{2}} f_{\boldsymbol{k}+\frac{q}{2}}^{\mathrm{a}} h_{k-\frac{q}{2}}^{\mathrm{r}}\right) \\
& \left.+\left(a_{j}^{\alpha} a_{k}^{\beta}+a_{k}^{\alpha} a_{j}^{\beta}\right) \hat{\gamma}_{\boldsymbol{k}-\frac{q}{2}}^{\alpha} \hat{\gamma}_{k+\frac{q}{2}}^{\beta} h_{k-\frac{q}{2}}^{\mathrm{r}} h_{k+\frac{q}{2}}^{\mathrm{a}}\right] .
\end{aligned}
$$

The antisymmetric component induced by the chiral nature is

$$
\sigma_{j k}^{\mathrm{a}}(\boldsymbol{q})=2 i \sum_{\boldsymbol{k}}\left[-\frac{1}{m}\left(k_{j} \boldsymbol{a}_{k}-k_{k} \boldsymbol{a}_{j}\right) \cdot\left(\hat{\boldsymbol{\gamma}}_{\boldsymbol{k}-\frac{q}{2}} \times \hat{\boldsymbol{\gamma}}_{\boldsymbol{k}+\frac{q}{2}}\right) h_{\boldsymbol{k}-\frac{q}{2}}^{\mathrm{r}} h_{\boldsymbol{k}+\frac{q}{2}}^{\mathrm{a}}+\left(\boldsymbol{a}_{j} \times \boldsymbol{a}_{k}\right) \cdot\left(\hat{\boldsymbol{\gamma}}_{\boldsymbol{k}+\frac{q}{2}} f_{\boldsymbol{k}-\frac{q}{2}}^{\mathrm{r}} h_{k+\frac{q}{2}}^{\mathrm{a}}-\hat{\boldsymbol{\gamma}}_{\boldsymbol{k}-\frac{q}{2}} f_{\boldsymbol{k}+\frac{q}{2}}^{\mathrm{a}} h_{\boldsymbol{k}-\frac{q}{2}}^{\mathrm{r}}\right)\right] .
$$

We see that the leading order is linear in $\boldsymbol{q}$. Using $\left(\boldsymbol{a}_{j} \times \boldsymbol{a}_{k}\right) \cdot \boldsymbol{\gamma}_{\boldsymbol{k}}=\lambda^{2} \epsilon_{j k l} \hat{\boldsymbol{k}}_{l}$ for the Weyl case,

$$
\sigma_{j k}^{\mathrm{a}}=\sigma_{0}^{\mathrm{a}} \epsilon_{j k l} q_{l},
$$

where $\sigma_{0}^{\mathrm{a}}$ is a finite constant. This component induces a chiral surface current in the plane perpendicular to the applied electric field, called the anomalous edge current in Ref. [15].

For the Rashba case, $\left(\boldsymbol{a}_{j} \times \boldsymbol{a}_{k}\right) \cdot \boldsymbol{\gamma}_{\boldsymbol{k}}=0,\left(\boldsymbol{a}_{j} \times \boldsymbol{a}_{k}\right) \cdot \boldsymbol{a}_{l}=0$, and the asymmetric component vanishes.

\section{APPENDIX C: INDUCED SPIN}

The induced spin due to the applied electric field is represented by a correlation function

$$
s_{j k}(\boldsymbol{q})=\frac{-1}{\Omega} \int \frac{d \omega}{2 \pi} \sum_{\boldsymbol{k}} \operatorname{tr}\left[\sigma_{j} G_{\boldsymbol{k} \omega} v_{k} G_{\boldsymbol{k}+\boldsymbol{q}, \omega+\Omega}\right]^{<} .
$$

We consider the uniform component, which is

$$
s_{j k}(0)=\sum_{k} \operatorname{tr}\left[\sigma_{j}\left(\frac{k_{k}}{m}\left[f_{k}^{\mathrm{r}} h_{\boldsymbol{k}}^{\mathrm{a}}\left(\hat{\gamma}_{\boldsymbol{k}} \cdot \boldsymbol{\sigma}\right)+\text { c.c. }\right]+a_{k}^{l}\left[\sigma_{l} f_{\boldsymbol{k}}^{\mathrm{r}} f_{\boldsymbol{k}}^{\mathrm{a}}+h_{\boldsymbol{k}}^{\mathrm{r}} h_{\boldsymbol{k}}^{\mathrm{a}}\left(\hat{\gamma}_{\boldsymbol{k}} \cdot \boldsymbol{\sigma}\right) \sigma_{l}\left(\hat{\boldsymbol{\gamma}}_{\boldsymbol{k}} \cdot \boldsymbol{\sigma}\right)\right]\right)\right] \text {. }
$$

For the Weyl case,

$$
s_{j k}(0)=2 \delta_{j k} \sum_{k}\left[\frac{k}{3 m}\left(f_{k}^{\mathrm{r}} h_{k}^{\mathrm{a}}+\text { c.c. }\right)+\lambda f_{k}^{\mathrm{r}} f_{k}^{\mathrm{a}}-\frac{\lambda}{3} h_{k}^{\mathrm{r}} h_{k}^{\mathrm{a}}\right]
$$

\section{APPENDIX D: EVALUATION OF SUMMATION OVER $k$ in $c$ and $\eta^{\mathrm{a}}$}

Two chiral coefficients of our interest are

$$
\begin{aligned}
c= & -\frac{4}{3} \lambda^{2} \frac{e}{V} \sum_{k} k \operatorname{Im}\left(f_{k}^{\mathrm{r}} h_{k}^{\mathrm{a}}\right) \\
\eta^{\mathrm{a}}= & -\frac{\lambda^{2}}{3 m} \operatorname{Im} \sum_{k} k^{2}\left[\frac{1}{\gamma_{k}} f_{k}^{\mathrm{r}} h_{k}^{\mathrm{a}}+\left(f_{k}^{\mathrm{r}} f_{k}^{\mathrm{a}(2)}-h_{k}^{\mathrm{r}} h_{k}^{\mathrm{a}(2)}\right.\right. \\
& \left.\left.+\frac{m \lambda}{k}\left(f_{k}^{\mathrm{r}} h_{k}^{\mathrm{a}(2)}-h_{k}^{\mathrm{r}} f_{k}^{\mathrm{a}(2)}\right)\right)\right] .
\end{aligned}
$$

In terms of the diagonalized Green's function,

$$
\begin{aligned}
\operatorname{Im}\left(f_{\boldsymbol{k}}^{\mathrm{r}} h_{\boldsymbol{k}}^{\mathrm{a}}\right) & =-\frac{1}{2} \operatorname{Im}\left[g_{\boldsymbol{k}+}^{\mathrm{r}} g_{\boldsymbol{k}-}^{\mathrm{a}}\right], \\
\operatorname{Im}\left(f_{\boldsymbol{k}}^{\mathrm{r}} f_{\boldsymbol{k}}^{\mathrm{a}(2)}\right) & =\frac{1}{4} \sum_{\sigma} \operatorname{Im}\left[g_{\boldsymbol{k} \sigma}^{\mathrm{r}}\left(g_{\boldsymbol{k} \sigma}^{\mathrm{a}}\right)^{2}+g_{\boldsymbol{k} \sigma}^{\mathrm{r}}\left(g_{\boldsymbol{k},-\sigma}^{\mathrm{a}}\right)^{2}\right], \\
\operatorname{Im}\left(h_{k}^{\mathrm{r}} h_{k}^{\mathrm{a}(2)}\right) & =\frac{1}{4} \sum_{\sigma} \operatorname{Im}\left[g_{\boldsymbol{k} \sigma}^{\mathrm{r}}\left(g_{\boldsymbol{k} \sigma}^{\mathrm{a}}\right)^{2}-g_{\boldsymbol{k} \sigma}^{\mathrm{r}}\left(g_{\boldsymbol{k},-\sigma}^{\mathrm{a}}\right)^{2}\right], \\
\operatorname{Im}\left(f_{\boldsymbol{k}}^{\mathrm{r}} f_{\boldsymbol{k}}^{\mathrm{a}(2)}-h_{\boldsymbol{k}}^{\mathrm{r}} h_{k}^{\mathrm{a}(2)}\right) & =\frac{1}{2} \sum_{\sigma} \operatorname{Im}\left[g_{\boldsymbol{k} \sigma}^{\mathrm{r}}\left(g_{\boldsymbol{k},-\sigma}^{\mathrm{a}}\right)^{2}\right] .
\end{aligned}
$$


The summation over $\boldsymbol{k}$ is carried out with use of contour integration. Using the density of states, $v(\epsilon) \propto \sqrt{\epsilon+\epsilon_{F}}$,

$$
\begin{aligned}
\sum_{\boldsymbol{k}} g_{\boldsymbol{k}+}^{\mathrm{r}} g_{\boldsymbol{k}-}^{\mathrm{a}} & =\int d \epsilon \nu(\epsilon) \frac{1}{\epsilon+\gamma_{\epsilon}-\frac{i}{2 \tau}} \frac{1}{\epsilon-\gamma_{\epsilon}+\frac{i}{2 \tau}} \\
& =-\frac{\pi}{2} i \sum_{\sigma} \frac{v_{\sigma}}{\gamma_{\sigma}-\frac{i}{2 \tau}}, \\
\sum_{\boldsymbol{k}} g_{k+}^{\mathrm{r}}\left(g_{\boldsymbol{k}-}^{\mathrm{a}}\right)^{2} & =-\frac{\pi}{4} i\left[\sum_{\sigma} \frac{v_{\sigma}}{\left(\gamma_{\sigma}-\frac{i}{2 \tau}\right)^{2}}-\frac{\nu_{-}}{\gamma_{-}-\frac{i}{2 \tau}} \frac{1}{2 \epsilon_{-}}\right],
\end{aligned}
$$

where $\nu_{\sigma}, \epsilon_{\sigma}$, and $\gamma_{\sigma}$ denote the density of states, energy $\epsilon$, and $\gamma_{k}$ evaluated at $k_{\sigma}$, i.e., the Fermi wave vector of the spin $\sigma$ state $\left[k_{\sigma} \equiv \sqrt{2 m\left(\epsilon_{F}+\sigma \gamma_{\sigma}\right)}\right.$ and $\left.\epsilon_{\sigma}=\epsilon_{F}+\sigma \gamma_{\sigma}\right]$. The imaginary parts are

$$
\begin{aligned}
& \operatorname{Im} \sum_{\boldsymbol{k}} g_{\boldsymbol{k}+}^{\mathrm{r}} g_{\boldsymbol{k}-}^{\mathrm{a}} \\
& \quad=-\frac{\pi}{2} \sum_{\sigma} \frac{v_{\sigma} \gamma_{\sigma}}{\left(\gamma_{\sigma}\right)^{2}+\frac{1}{4 \tau^{2}}}, \\
& \operatorname{Im} \sum_{\boldsymbol{k}} g_{\boldsymbol{k}+}^{\mathrm{r}}\left(g_{\boldsymbol{k}-}^{\mathrm{a}}\right)^{2} \\
& =-\frac{\pi}{4}\left[\sum_{\sigma} \frac{v_{\sigma}\left[\left(\gamma_{\sigma}\right)^{2}-\frac{1}{4 \tau^{2}}\right]}{\left[\left(\gamma_{\sigma}\right)^{2}+\frac{1}{4 \tau^{2}}\right]^{2}}-\frac{\nu_{-}}{\left(\gamma_{-}\right)^{2}+\frac{1}{4 \tau^{2}}} \frac{\gamma_{-}}{\epsilon_{-}}\right] .
\end{aligned}
$$

The chiral coefficients are thus

$$
\begin{aligned}
c= & -\frac{\pi}{3} \frac{e \lambda^{2}}{a^{3}} \sum_{\sigma} \frac{v_{\sigma} \gamma_{\sigma} k_{\sigma}}{\left(\gamma_{\sigma}\right)^{2}+\frac{1}{4 \tau^{2}}}, \\
\eta^{\mathrm{a}}= & -\frac{\pi e \lambda^{2}}{24 m a^{3}} \sum_{\sigma} v_{\sigma} k_{\sigma}^{2}\left[\frac{1}{\tau^{2}} \frac{1}{\left[\left(\gamma_{\sigma}\right)^{2}+\frac{1}{4 \tau^{2}}\right]^{2}}\right. \\
& \left.+2 \frac{\gamma_{\sigma}}{\epsilon_{\sigma}} \frac{1}{\left(\gamma_{\sigma}\right)^{2}+\frac{1}{4 \tau^{2}}}\left(1+\sigma \frac{m \lambda}{2 k_{\sigma}}\right)\right] .
\end{aligned}
$$

The limit value for the clean $\left(\gamma_{\sigma} \tau \gg 1\right)$ limit is

$$
\begin{aligned}
c & \simeq-\frac{\pi}{3} \frac{e \lambda^{2}}{a^{3}} \sum_{\sigma} \frac{v_{\sigma} k_{\sigma}}{\gamma_{\sigma}} \\
\eta^{\mathrm{a}} & \simeq-\frac{\pi e \lambda^{2}}{24 m a^{3}} \sum_{\sigma} v_{\sigma} k_{\sigma}^{2} \frac{1}{\gamma_{\sigma} \epsilon_{\sigma}}\left(1+\sigma \frac{m \lambda}{2 k_{\sigma}}\right)
\end{aligned}
$$

while

$$
\begin{aligned}
c & \simeq-\frac{4 \pi}{3} \frac{e \lambda^{2}}{a^{3}} \sum_{\sigma} v_{\sigma} \gamma_{\sigma} k_{\sigma} \tau^{2} \\
\eta^{\mathrm{a}} & \simeq-\frac{2 \pi e \lambda^{2}}{3 m a^{3}} \sum_{\sigma} v_{\sigma} k_{\sigma}^{2} \tau^{2}\left[1+\frac{\gamma_{\sigma}}{2 \epsilon_{\sigma}}\left(1+\sigma \frac{m \lambda}{2 k_{\sigma}}\right)\right]
\end{aligned}
$$

for the dirty $\left(\gamma_{\sigma} \tau \ll 1\right)$ limit.

The spin density response function is

$$
\begin{aligned}
\kappa_{s} & =2 \frac{e}{V} \sum_{k} \operatorname{Re}\left[\frac{2 k}{3 m} f_{k}^{\mathrm{r}} h_{k}^{\mathrm{a}}+\lambda f_{k}^{\mathrm{r}} f_{k}^{\mathrm{a}}-\frac{\lambda}{3} h_{k}^{\mathrm{r}} h_{k}^{\mathrm{a}}\right] \\
& =\frac{2 \pi e}{3 a^{3}} \sum_{\sigma} \frac{v_{\sigma}}{k_{\sigma}} \tau\left(\sigma \epsilon_{\sigma}+\gamma_{\sigma} \frac{\gamma_{\sigma}^{2}+\frac{3}{8 \tau^{2}}}{\gamma_{\sigma}^{2}+\frac{1}{4 \tau^{2}}}\right) .
\end{aligned}
$$

\section{APPENDIX E: RELAXATION FORCE DUE TO IMPURITY SCATTERING}

In this Appendix, we discuss the relaxation force due to impurity scattering descried by a Hamiltonian

$$
H_{\mathrm{i}}=\sum_{\boldsymbol{k} \boldsymbol{k}^{\prime}} u_{\boldsymbol{k}^{\prime}-\boldsymbol{k}} c_{\boldsymbol{k}^{\prime}}^{\dagger} c_{\boldsymbol{k}},
$$

where $u_{\boldsymbol{k}^{\prime}-\boldsymbol{k}}=u_{\mathrm{i}} \sum_{\boldsymbol{R}_{i}} e^{-i\left(\boldsymbol{k}^{\prime}-\boldsymbol{k}\right) \cdot \boldsymbol{R}_{i}}$ is an impurity potential, where $u_{\mathrm{i}}$ is the strength of the impurity potential and $\boldsymbol{R}_{i}$ is the position of the $i$ th impurity. Chiral effects are not considered. As the linear response to the applied electric field, the uniform component of the relaxation force due to the impurity scattering is

$$
\boldsymbol{f}_{\text {rel }}=2 e \sum_{\boldsymbol{k} \boldsymbol{k}^{\prime}} i u_{\mathrm{i}}^{2} \boldsymbol{k}\left(\frac{\boldsymbol{k}}{m} \cdot \boldsymbol{E}\right)\left(g_{\boldsymbol{k}^{\prime}}^{\mathrm{a}}-g_{\boldsymbol{k}^{\prime}}^{\mathrm{r}}\right) g_{\boldsymbol{k}}^{\mathrm{a}} g_{\boldsymbol{k}}^{\mathrm{r}}=-e \frac{4}{3} v \epsilon_{\mathrm{F}} \boldsymbol{E} .
$$

Using the electron density $n=\frac{4}{3} \nu \epsilon_{\mathrm{F}}$, the relaxation force is $\boldsymbol{f}_{\text {rel }}=-e n \boldsymbol{E}$. It therefore cancels the direct driving force due to the applied field in the present static case. Using the current $\boldsymbol{j}=\frac{n e^{2} \tau}{m} \boldsymbol{E}$, the force is represent as $\boldsymbol{f}_{\text {rel }}=-\frac{1}{\tau} \frac{m}{e} \boldsymbol{j}$.
[1] L. D. Landau and E. M. Lifshitz, Fluid Mechanics, 2nd ed. (Butterworth-Heinemann, Oxford, 1987).

[2] K. Kruse, J. F. Joanny, F. Jülicher, J. Prost, and K. Sekimoto, Generic theory of active polar gels: A paradigm for cytoskeletal dynamics, Eur. Phys. J. E 16, 5 (2005).

[3] S. Fürthauer, M. Strempel, S. W. Grill, and F. Jülicher, Active chiral fluids, Eur. Phys. J. E 35, 89 (2012).

[4] T. Markovich, E. Tjhung, and M. E. Cates, Chiral active matter: Microscopic 'torque dipoles' have more than one hydrodynamic description, New J. Phys. 21, 112001 (2019).

[5] A. Lucas and K. C. Fong, Hydrodynamics of electrons in graphene, J. Phys.: Condens. Matter 30, 053001 (2018).
[6] B. N. Narozhny, Electronic hydrodynamics in graphene, Ann. Phys. 411, 167979 (2019).

[7] I. Torre, A. Tomadin, A. K. Geim, and M. Polini, Nonlocal transport and the hydrodynamic shear viscosity in graphene, Phys. Rev. B 92, 165433 (2015).

[8] D. A. Bandurin, I. Torre, R. K. Kumar, M. Ben Shalom, A. Tomadin, A. Principi, G. H. Auton, E. Khestanova, K. S. Novoselov, I. V. Grigorieva, L. A. Ponomarenko, A. K. Geim, and M. Polini, Negative local resistance caused by viscous electron backflow in graphene, Science 351, 1055 (2016).

[9] L. Levitov and G. Falkovich, Electron viscosity, current vortices and negative nonlocal resistance in graphene, Nat. Phys. 12, 672 (2016). 
[10] R. Gurzhi, Minimum of resistance in impurity-free conductors, JETP 17, 521 (1963) [Zh. Eksp. Teor. Fiz. 44, 771 (1963)].

[11] J. A. Sulpizio, L. Ella, A. Rozen, J. Birkbeck, D. J. Perello, D. Dutta, M. Ben-Shalom, T. Taniguchi, K. Watanabe, T. Holder, R. Queiroz, A. Principi, A. Stern, T. Scaffidi, A. K. Geim, and S. Ilani, Visualizing poiseuille flow of hydrodynamic electrons, Nature 576, 75 (2019).

[12] B. N. Narozhny, I. V. Gornyi, M. Titov, M. Schütt, and A. D. Mirlin, Hydrodynamics in graphene: Linear-response transport, Phys. Rev. B 91, 035414 (2015).

[13] A. Lucas and S. A. Hartnoll, Kinetic theory of transport for inhomogeneous electron fluids, Phys. Rev. B 97, 045105 (2018).

[14] M.-C. Chang and Q. Niu, Berry phase, hyperorbits, and the Hofstadter spectrum: Semiclassical dynamics in magnetic Bloch bands, Phys. Rev. B 53, 7010 (1996).

[15] R. Toshio, K. Takasan, and N. Kawakami, Anomalous hydrodynamic transport in interacting noncentrosymmetric metals, Phys. Rev. Res. 2, 032021(R) (2020).

[16] S. Conti and G. Vignale, Elasticity of an electron liquid, Phys. Rev. B 60, 7966 (1999).

[17] X. Gao, J. Tao, G. Vignale, and I. V. Tokatly, Continuum mechanics for quantum many-body systems: Linear response regime, Phys. Rev. B 81, 195106 (2010).

[18] A. Principi, G. Vignale, M. Carrega, and M. Polini, Bulk and shear viscosities of the two-dimensional electron liquid in a doped graphene sheet, Phys. Rev. B 93, 125410 (2016).
[19] P. A. Frigeri, Superconductivity in crystals without an inversion center, Ph.D. thesis, ETH-Zurich, 2005

[20] J. Kang and J. Zang, Transport theory of metallic $b 20$ helimagnets, Phys. Rev. B 91, 134401 (2015).

[21] H. Nielsen and M. Ninomiya, Absence of neutrinos on a lattice: (i). proof by homotopy theory, Nucl. Phys. B 185, 20 (1981).

[22] M. M. Vazifeh and M. Franz, Electromagnetic Response of Weyl Semimetals, Phys. Rev. Lett. 111, 027201 (2013).

[23] M. Hirayama, R. Okugawa, S. Ishibashi, S. Murakami, and T. Miyake, Weyl node and spin texture in trigonal tellurium and selenium, Phys. Rev. Lett. 114, 206401 (2015).

[24] J. Rammer, Quantum Field Theory of Non-Equilibrium States (Cambridge University Press, Cambridge, 2007).

[25] R. Naaman and D. H. Waldeck, Spintronics and chirality: Spin selectivity in electron transport through chiral molecules, Annu. Rev. Phys. Chem. 66, 263 (2015).

[26] T. Yoda, T. Yokoyama, and S. Murakami, Orbital edelstein effect as a condensed-matter analog of solenoids, Nano Lett. 18, 916 (2018).

[27] L. D. Barron, From cosmic chirality to protein structure: Lord kelvin's legacy, Chirality 24, 879 (2012).

[28] X.-L. Qi and S.-C. Zhang, Topological insulators and superconductors, Rev. Mod. Phys. 83, 1057 (2011).

[29] H. Kawaguchi and G. Tatara, Effective hamiltonian approach to optical activity in weyl spin-orbit system, J. Phys. Soc. Jpn. 87, 064002 (2018). 